\title{
Investigations on PD Characteristics of Thermal aged Palm and Corn Oil for Power Transformer Insulation Applications
}

\begin{abstract}
S.Senthilkumar", B.Karthik"* and S.Chandrasekar ${ }^{\dagger}$
Abstract - Partial discharge (PD) detection plays a major role in the life time assessment of liquid insulation in power transformers. Many research works are being carried out to replace conventional mineral oil insulation in transformers by vegetable oils. It is necessary to understand the PD characteristics of vegetable oils before recommending them as an alternate for mineral oil. In this paper, the breakdown strength and PD characteristics of palm and corn oil were investigated in both unaged and thermally aged conditions. Laboratory experiments were performed as per IEC test procedures. PD signals were measured using wide band detection system. Phase resolved PD pattern of vegetable oils and mineral oil were compared. Effect of increase in voltage stress on the PD pattern of palm and corn oil were studied. Time and frequency domain analysis of PD pulses at needle-plane electrode configuration was carried out. Statistical analysis of PD pattern i.e. skewness and shape parameter variations with respect to applied thermal stress were also carried out. From the results, it is observed that palm and corn oils have better breakdown strength and PD characteristics even under long-term thermal stress and hence they can be used for power transformer applications.
\end{abstract}

Keywords: Power transformer, Breakdown strength, Partial discharge, Vegetable oil, Frequency spectrum, Statistical analysis.

\section{Introduction}

Mineral oils are the most commonly used fluids for electrical insulation and heat transfer in electric power apparatus and systems [1]. The major problem faced with mineral oil is that it is poorly biodegradable and could cause serious contamination of soil and waterways if spills occur. In addition, it is estimated that petroleum products are eventually going to run out leading to serious shortages of mineral oil by the mid-twenty first century. Hence, it is necessary to carryout extensive studies in the development of new biodegradable insulating fluids. Vegetable oils are natural products and are plenty of supply. The most attractive features of vegetable oils are that they are biodegradable, non-toxic and most environmental friendly. Extensive studies were carried out for the past two decades to find a suitable alternate vegetable oil for electrical applications [2-14]. The major problem faced with the liquid insulation for transformer applications is the aging and degradation of the insulating medium due to various stresses such as electrical and thermal, which in turn affects the long-term performance of the oil. There is now considerable interest in understanding the thermal aging characteristics of vegetable oils which is not yet well

$\dagger$ Corresponding Author: Dept. of Electrical and Electronics Engineering, Gnanamani College of Technology, India. (chandrukvt@ gmail.com)

* Dept. of Mechanical Engineering, R.M.K. College of Engg. and Tech., Puduvoyal, India. (s.senthilkumarph.d@gmail.com)

** Dept. of Electrical and Electronics Engineering, Sona College of Technology, India. (karthik pse@yahoo.co.in)

Received: October 27, 2013; Accepted: March 3, 2014 reported [8-11].

Partial discharge (PD) due to electric field enhancement in a localized area of insulation accelerates the degradation and thermal aging of the insulating oil. Hence it plays a major role in determining the insulation strength and life time of oil. Therefore, it is important to understand the PD characteristics of vegetable oils before recommending them as an alternate of mineral oil for high voltage transformer applications. PD detection and analysis is now a well recognized insulation monitoring method in determining the quality of insulation. However, the PD characteristics of vegetable oils such as corn oil and palm oil under unaged and thermally aged conditions are scanty.

Considering these facts, the major aim of the present work is to collect the typical PD patterns from palm and corn oils in order to find its suitability for power apparatus applications. In this paper, phase resolved PD characteristics and breakdown strength characteristics of corn oil and palm oil under unaged and thermally aged conditions are investigated. For comparison purpose, the PD characteristics of mineral oil are also discussed. Time and frequency domain analysis of PD pulses are discussed. Statistical analysis of PD pattern i.e. skewness and shape parameter variations with respect to applied thermal stress are also carried out.

\section{Experimental Procedures}

Commercially available corn oil with free fatty acid 
Table 1. Properties of oils as per manufacturer's data

\begin{tabular}{c|c|c|c|c|c|c|c}
\hline & $\mathrm{FP} 1{ }^{\circ} \mathrm{C}$ & $\mathrm{FP} 2{ }^{\circ} \mathrm{C}$ & $\begin{array}{c}\mathrm{V} \\
@ 400^{\circ} \mathrm{C} \\
\mathrm{mm}^{2} / \mathrm{s}\end{array}$ & $\mathrm{M} \%$ & SFA \% & $\begin{array}{c}\text { MUSFA } \\
\%\end{array}$ & $\begin{array}{c}\mathrm{PU} \\
\text { SFA \% }\end{array}$ \\
\hline $\mathrm{C}$ & 305 & 350 & 31 & 0.3 & 13.5 & 25.6 & 60.8 \\
\hline $\mathrm{P}$ & 250 & 260 & 37 & 0.12 & 50 & 39.5 & 10.5 \\
\hline $\mathrm{M}$ & 145 & 160 & 14 & 0.1 & -- & -- & -- \\
\hline
\end{tabular}

C-corn, P-palm, M-mineral, FP1-Flash point, FP2-Fire point, V-Viscosity, M-Moisture, SFA-Saturated fatty acid, MUSFA-Monounsaturated fatty acid, PUSFA-polyunsaturated fatty acid

(FFA) of $3 \%$ and palm oil with FFA of $1 \%$ supplied by GR brand were used in the present study. Commercially available TRANSOL brand mineral oil was used for the experimental studies and the characteristics of oils are given in Table 1. Initially, in order to remove any moisture content present, oil sample was kept in a sealed steel container and thermally treated at $70^{\circ} \mathrm{C}$ for 48 hours inside a temperature controlled oven and then the sample was allowed to cool to room temperature for 24 hours. Accelerated thermal aging was performed in the laboratory to expose the oil to extreme temperature conditions for a certain period of time in order to understand the change in dielectric characteristics at elevated temperatures. In this thermal aging process, oil samples were kept in sealed steel containers and placed inside a temperature controlled oven at $150^{\circ} \mathrm{C}$ and taken out after 15 days, 30 days and 45 days. In order to understand the influence of transformer windings and its catalytic effects during the thermal aging, oil sample was also added with copper pieces $(1 \mathrm{~mm}$ diameter and $5 \mathrm{~cm}$ length) during aging process [8]. Unless otherwise mentioned, the results reported in this paper for thermally aged oil samples are without copper.

\subsection{Breakdown Voltage (BDV) Test}

The value of breakdown voltage gives the electrical strength of the insulating medium. This test was carried out as per IEC 60156 standard. Fig. 1 shows the schematic diagram of the experimental setup used to evaluate the breakdown voltage of oil samples. Initially the oil samples were filtered using a 5 micron filter paper in order to remove any impurities present in the oil. Test chamber, consisting of spherical electrodes of $12.5 \mathrm{~mm}$ diameter and separated by $2.5 \mathrm{~mm}$ gap distance, was filled with the

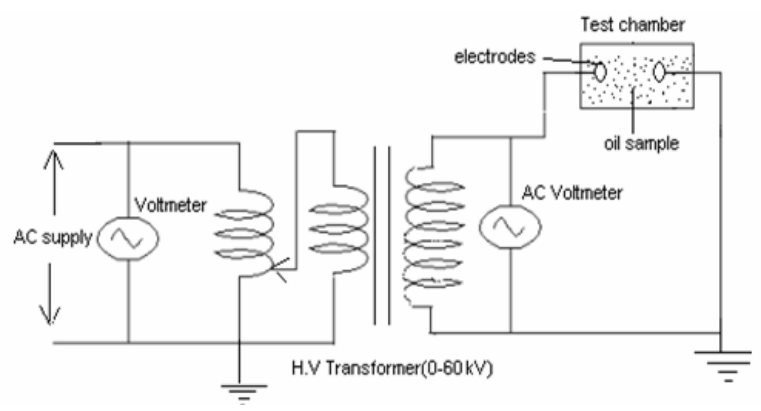

Fig. 1. Experimental setup for BDV test insulating oil. High voltage AC supply was slowly increased at a rate of $2 \mathrm{kV} / \mathrm{s}$ until the breakdown occurs. A time delay of 1 minute is used between successive breakdowns. This test was carried out for both virgin and thermally aged oil samples, at different electrode gap distances such as 1, 2, 2.5 and $3 \mathrm{~mm}$ and corresponding breakdown voltage was noted. Eight breakdowns on the same specimen were carried out and the average value was used as the electrical strength of insulation oil.

\subsection{Partial Discharge test}

A partial discharge in a high voltage transformer occurs when the electric field enhances in a localized area of insulation. Partial discharge detection is one of the most important diagnostic techniques used to assess condition of high voltage insulation in transformer. The most likely types of PDs in a transformer can be broken down into two categories such as corona discharges and surface discharges. Corona discharge may be due to any floating conducting particle in the insulating medium. Surface discharges in transformers mainly occur in the surface of pressboard layers.

In the present work, in order to simulate corona discharge, a needle-plane electrode configuration was used. Partial discharge measurement is carried out according to IEC 60270 test procedures. Fig. 2 shows the test cell with needle-plane electrode configuration. The tip of the needle has a curvature radius of $1.5 \mu \mathrm{m}$. The test specimen is energized from a step up transformer which can supply voltage upto $100 \mathrm{kV}$. Primary side of the high voltage transformer is connected to a control panel and a $230 \mathrm{~V}$ AC supply. The high voltage was connected to the needle electrode and the plane electrode was solidly grounded. Since it is difficult to get a stable PD source from the needle-plane electrode configuration, a $3 \mathrm{~mm}$ thick pressboard was used upon the ground electrode. The gap distance between the needle and ground electrode was maintained at $5 \mathrm{~mm}$.

PD signals were picked by connecting a high frequency current transformer (HFCT) around the ground connection of the test cell. HFCT is a clip on device clamped around the ground lead and it has a $50 \mathrm{MHz}$ frequency bandwidth which is sufficient to cover the entire range of PD. Output of the HFCT is connected to the PD detector. Partial discharges were detected through a large bandwidth system,

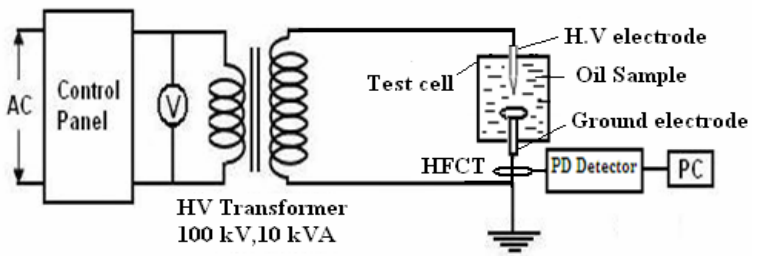

Fig. 2. Schematic diagram of the experimental setup for needle-plane electrode configuration PD test 
PDBASE II (TechImp Systems, Italy) able to sample the complete PD waveforms at a sampling rate of upto 100 $\mathrm{MSa} / \mathrm{s}$ and bandwidth of 0-50 MHz. The sensitivity ranges from $2 \mathrm{mV} /$ div to $5 \mathrm{~V} /$ div. PDBASE II also provides large number of digitized PD pulse waveforms and it is able to separate them according to the PD waveform shape [15-18]. The PD pulses were sent to a remote PC for further processing.

\section{Distribution Analysis of Breakdown Strength}

It is important to understand the correlation between the dispersion of breakdown voltages between the mineral oil and vegetable oils in order to estimate the safety margin while designing the insulation of electric power apparatus. Fig. 3 shows the breakdown voltage characteristics of oil samples at different gap distance of the electrodes with respect to thermal aging time. Minimum and maximum values of breakdown strength obtained during the experimental studies at each aging time period are also shown in Fig. 3. For comparison purpose, conventionally used mineral oil characteristics are also

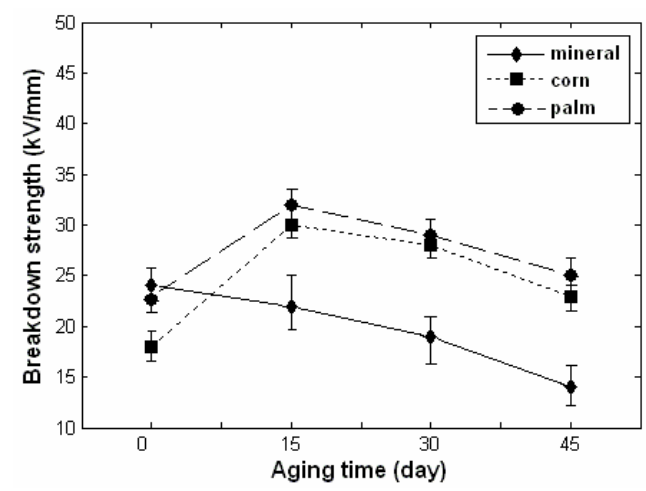

(a)

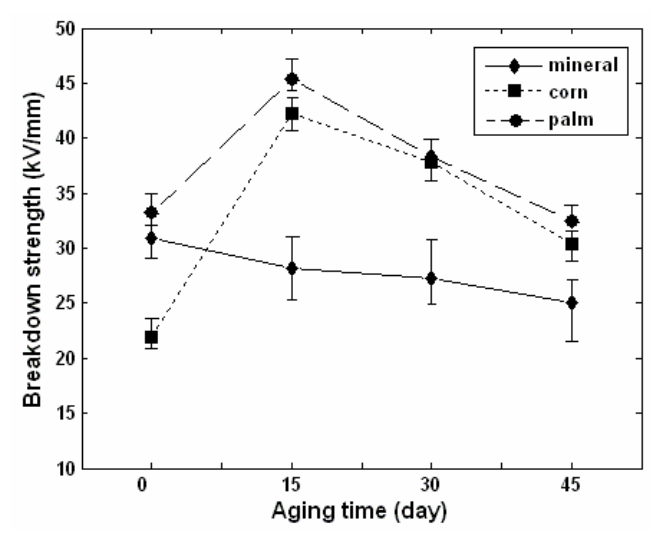

(b)

Fig. 3. Breakdown voltage values of insulation oil samples with respect to thermal aging time at different gap distance of electrodes: (a) $2 \mathrm{~mm}$; (b) $2.5 \mathrm{~mm}$ plotted.

Suwarno Aditama [6], have reported that increase in fat content considerably reduces the BDV value and palm oil with lower fat content showed BDV value more than $30 \mathrm{kV}$. The slight reduction in BDV value of corn oil may be due to the higher fat content compared with palm oil. With respect to increase in thermal aging time, the breakdown strength of mineral oil reduces considerably, whereas interestingly the palm oil and corn oil shows increase in breakdown strength after 15 days of thermal aging. This increase in BDV can only be attributed to a chemical change of the oil and similar results of increasing BDV after aging when compared with unaged oil is already reported [11]. After 30 days and 45 days of thermal aging, a steep reduction in breakdown strength of vegetable oils is noticed, the reason may be that due to long period of thermal stress, the fat in the oil due to oxidation may release volatile acid, aldehyde, water and may be also hydrogen gas. Increase in acid value and water content due to severe thermal aging and increase in oxidation products might have reduced the breakdown voltage [6]. However, decrease in breakdown strength of vegetable oil is relatively lower when compared with mineral oil. It may be due to the reason that vegetable oils make less sludge formation when react with oxygen [10]. In general, with respect to increase in gap distance between the electrodes, considerable increase in breakdown strength is noticed for all oil specimens.

Breakdown strength of a liquid dielectric medium is a statistical quantity which depends on the physico-chemical properties, impurities and particles present in the medium during test. Therefore statistical techniques have been extensively applied to understand the insulation failure.

D. Martin et al., [14] have reported that Weibull distribution tends to give closer results than the Gaussian distribution in the statistical analysis of the AC breakdown voltages of ester based transformer oils as it does not make assumptions of the skewness and kurtosis. Hence, in this work, two parameter Weibull distribution is used to fit the breakdown voltages. The Weibull cumulative distribution function, $F$, is defined as

$$
F\left(V_{b d}\right)=1-\exp \left[-\left(\frac{V_{b d}}{\alpha}\right)^{\beta}\right]
$$

where $\alpha$ is the scale parameter, which is the voltage at which $63 \%$ of the oil specimens have broken down and $\beta$ is the shape parameter, which is the Weibull modulus indicating the width of the distribution. MATLAB software was used to carry out Weibull analysis.

Fig. 4 shows the Weibull distribution of breakdown voltage characteristics of unaged oil samples, where the dotted line represents the fit to the data and the thick lines give $95 \%$ confidence intervals for these probabilities. Corresponding scale parameter $(\alpha)$ and shape parameter 

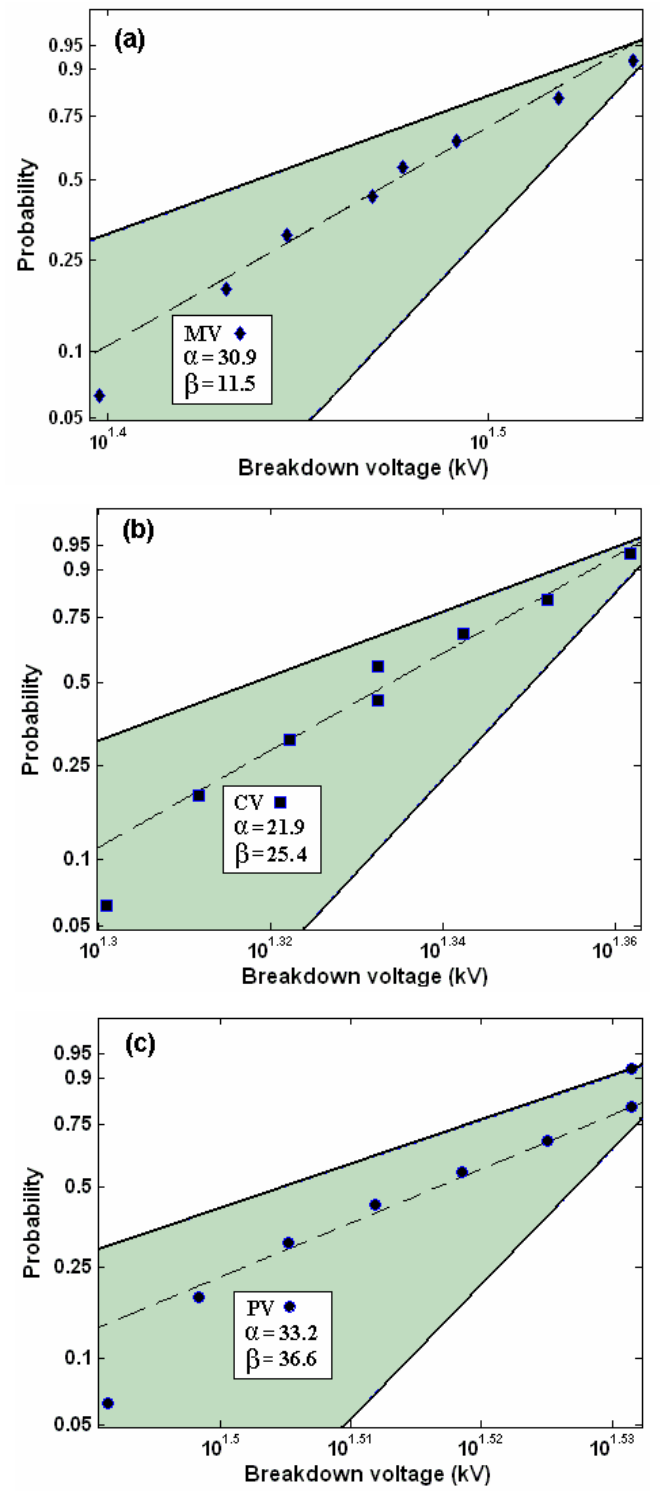

Fig. 4. Weibull distribution of breakdown voltage values of unaged oil samples at $2.5 \mathrm{~mm}$ gap distance of electrodes: (a) mineral oil; (b) corn oil; (c) palm oil

( $\beta$ ) strength of oil samples. The shape parameter $\beta$ is correlated with the breakdown strength dispersion. Shape parameter value of mineral oil lies below 15, whereas for both corn and palm oil lies above 25, which implies that palm and corn oils have smaller dispersion of breakdown voltages. Standard deviation of BDV data was calculated and found to be 1, 1.13 and 3 for palm, corn and mineral oil respectively and is in similar line withWeibull data analysis.

Fig. 5 show the variations in scale parameters of oil samples at different electrode gap values. In this plot, M, $\mathrm{P}$ and $\mathrm{C}$ stand for mineral oil, palm oil and corn oil respectively. Similarly, V, 30 and 45 stand for unaged, 30 days thermal aged, 45 days thermal aged respectively. It can be seen that, in general, palm and corn oil have better electrical breakdown strength and smaller breakdown

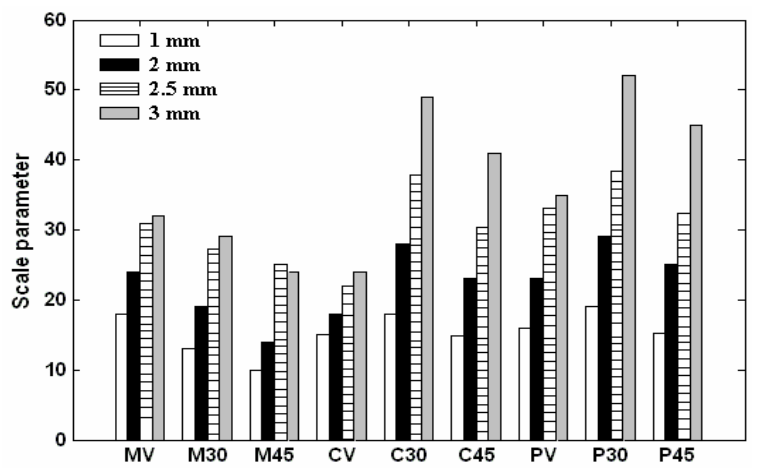

Fig. 5. Variations in scale parameter of oil samples at different electrode gaps, where $\mathrm{M}, \mathrm{P}$ and $\mathrm{C}$ stands for mineal oil, palm oil and corn oil respectively.

voltage data dispersion than mineral oil. In the case of mineral oil, thermal aging reduces considerably the scale parameter. However, even after 45 days of thermal stress, a slight reduction in scale parameter of palm oil and corn oil is noticed, but they still maintain BDV values close to 30 $\mathrm{kV}$ (2.5 mm gap).

In general, from the BDV test results it is noticed that palm and corn oils have the ability to retain the breakdown strength even under accelerated thermal aging for longer periods compared with conventionally used mineral oil. As discussed earlier, this may be due to the lowest turbidity and fat content between the molecules of the vegetable oils [6].

\section{Phase Resolved PD Pattern Analysis}

\subsection{PRPD pattern analysis}

In the needle-plane electrode configuration, initially the partial discharge inception voltage (PDIV) was evaluated. PDIV is the lowest voltage at which observable PD pulses appear on the PD detector. Pompili et al., [21] have reported that determination of PDIV in the case of liquid dielectrics is difficult because PD pulses appear in pulse bursts and their occurrence rate is more erratic.

PD signals were measured in stable condition after voltage application and a set of 5 PD measurements were carried out at each voltage level in order to extract the $95 \%$ of confidence level. Fig. 6 shows the PDIV of oil samples measured at $5 \mathrm{~mm}$ gap distance between the electrodes. In general, vegetable oils showed higher PDIV when compared with mineral oil. It is clear that thermal aging significantly reduced the PDIV. From the aged oil sample results, it is clear that palm and corn oil has slightly higher PDIV compared with mineral oil which may be attributed to the less sludge formation of vegetable oils even after accelerated thermal aging. It is also noticed that presence of copper during aging considerably reduced the PDIV. The reason may be that addition of copper to the oils 
catalyses the oxidation process and hence aging of oils is accelerated [8].

Once the PDIV is noted, then the test voltage was increased until a clear PD pattern of corona discharge is visible. In the case of PRPD pattern analysis peak discharge magnitude for each phase position window is

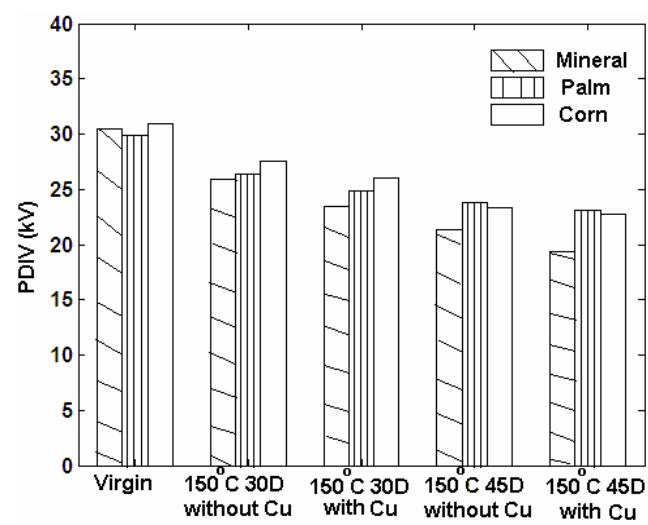

Fig. 6. Partial discharge inception voltage obtained at needle-plane electrode configuration of virgin, 30 days thermally aged and 45 days thermally aged oil with and without copper.

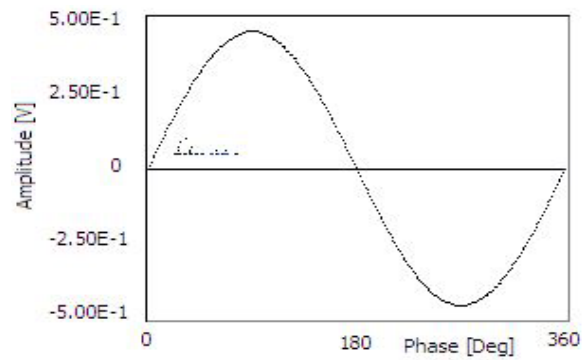

(i)

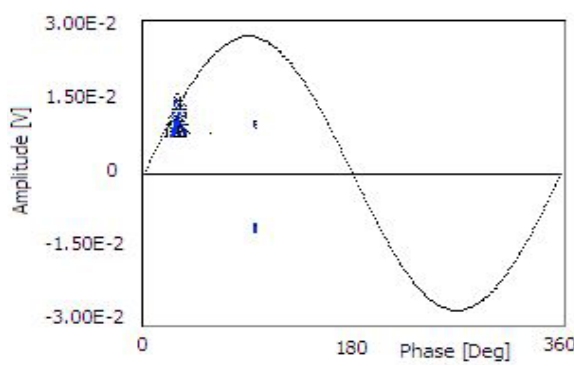

(i)

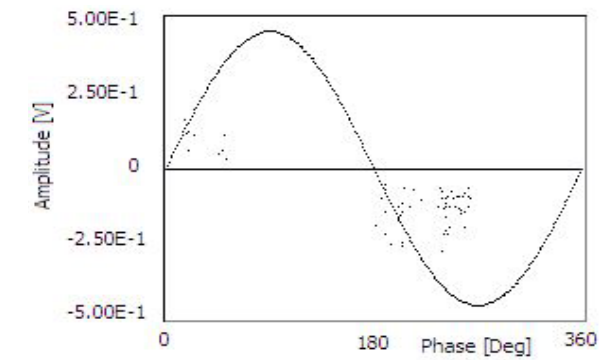

(ii)

(a)

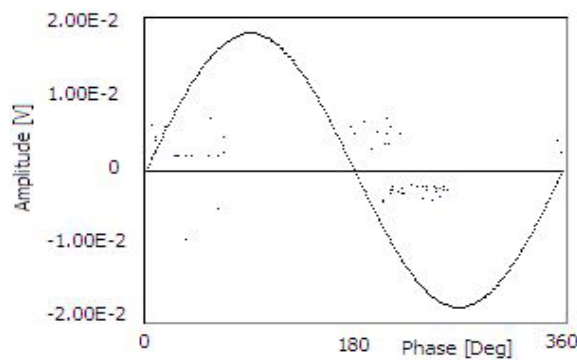

(b)

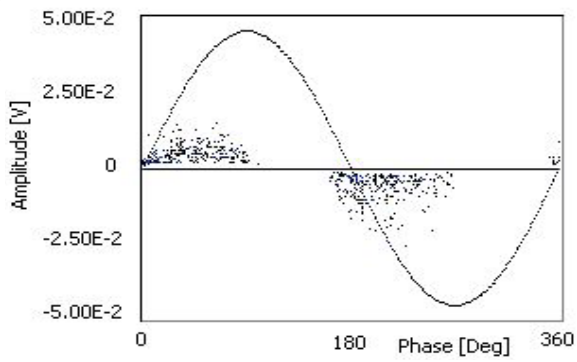

(ii)

(i)

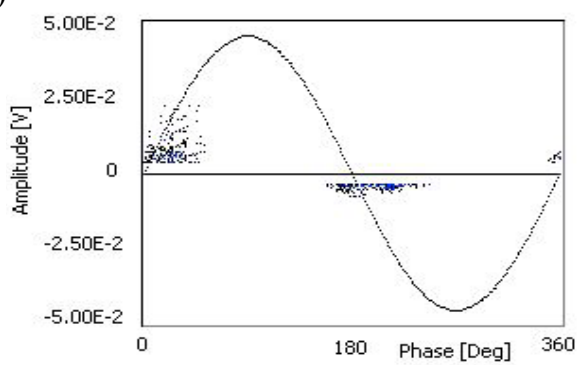

(ii)

(c)

Fig. 7. Typical partial discharge pattern obtained at needle-plane electrode configuration of virgin (noted as i) and 30 days thermal aged (noted as ii) oils: (a) mineral oil; (b) palm oil; (c) corn oil 

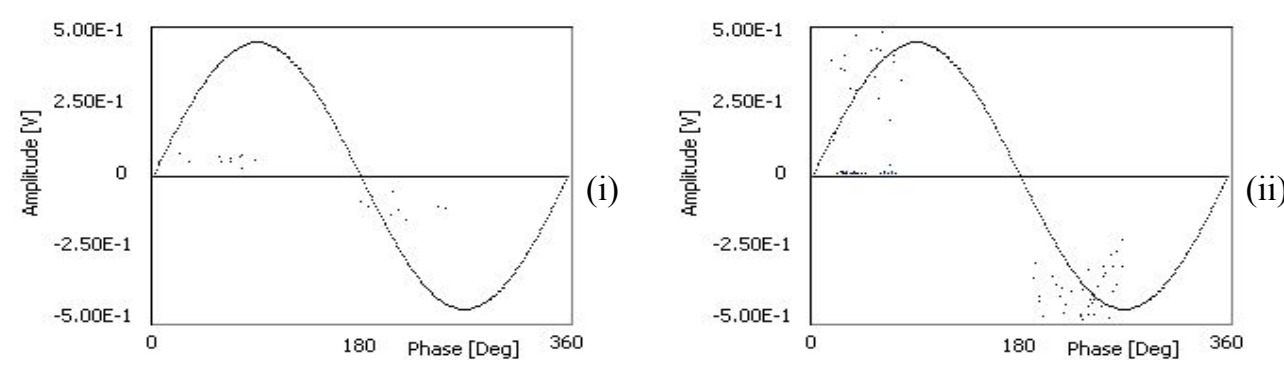

(a)
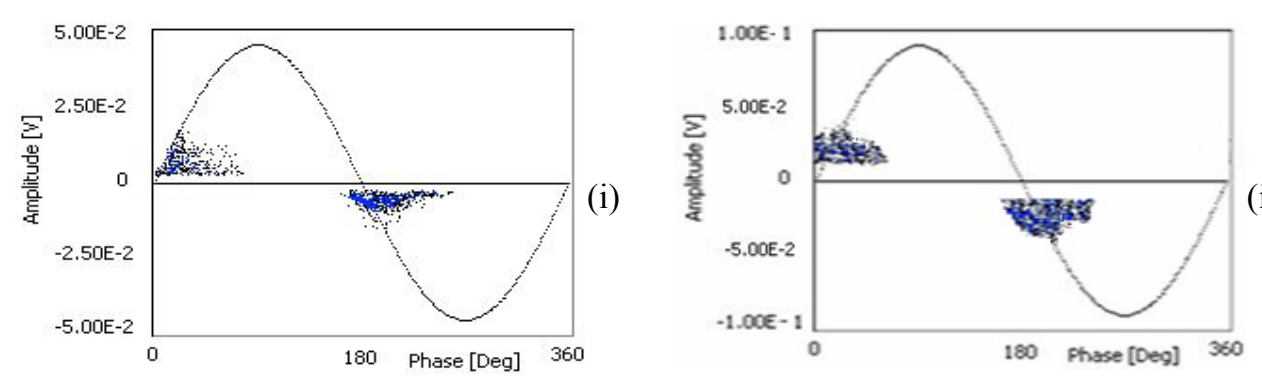

(ii)

(b)
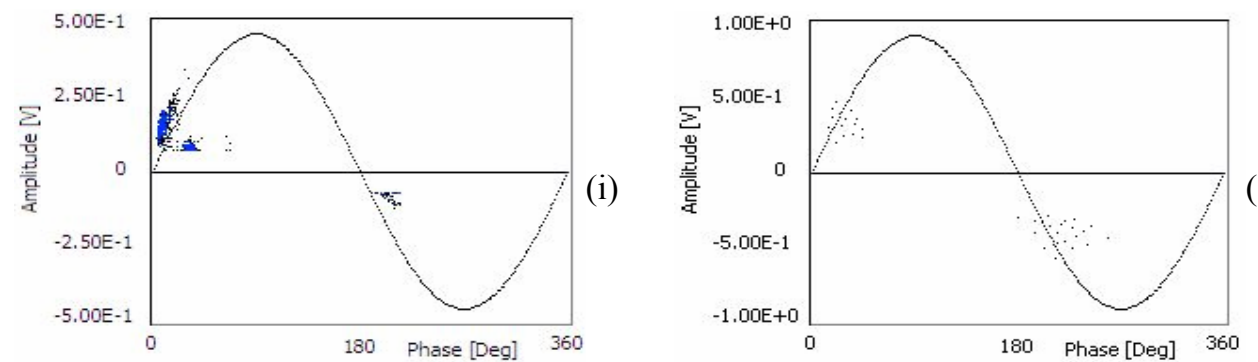

(c)

Fig. 8. Typical PD pattern obtained at needle-plane electrode configuration of 45 days thermal aged oil without copper (noted as i) and 45 days thermal aged oil with copper (noted as ii): (a) mineral; (b) palm; (c) corn

magnitude of mineral oil is higher when compared with vegetable oils. Since the amplitude of PD signal mainly depends on the voltage stress, it is important to understand the PD characteristics with respect to increase in test voltage for vegetable oils. Fig. 9 highlights the variations in PD amplitude and phase distortion with respect to increase in test voltage of both vegetable oils and mineral oil subjected to 30 days of thermal stress. In general, PD repetition rate of vegetable oils is higher compared with mineral oil. Since the vegetable oils are more hygroscopic than mineral oil, PD in vegetable oils propagate faster than mineral oil at the same voltage level and hence vegetable oils exhibit higher PD repetition rates under AC stress [21]. It is observed that in the case of mineral oil, the PD amplitude increases from $50 \mathrm{mV}$ to $500 \mathrm{mV}$ with increase in voltage, whereas PD amplitude in both palm and corn oil increases from $10 \mathrm{mV}$ to $40 \mathrm{mV}$, which is very less when compared with mineral oil. Initially, occurrence of PD at electric field enhancement sites in oil increases the local temperature, giving rise to the generation of fault gases such as ethane, hydrogen and acetylene. In earlier works, it is reported that generation rate of dissolved gases in vegetable oils is slightly lower than in mineral oils and vegetable oils are particularly stable under mediumtemperature thermal faults $[19,21]$. This may be the cause for the reduction in PD amplitude of vegetable oils. In the case of mineral oil, for the same amount of voltage stress, PDs with higher amplitudes are more effective in breakingup chemical bonds within liquid molecules and this may be the cause for the production of large concentrations of fault gases. In general, PD dispersion increases with increase in test voltage and it is considerably higher in the case of mineral oil. It is also observed that increase in applied voltage leads to decrease in PD phase inception delay irrespective of type of oil. From these test results, it is observed that the performance of palm and corn oil is slightly better when compared with conventionally used mineral oils.

\subsection{PD pulse time \& frequency domain analysis}

D.Imamovic et al., [20] have analyzed the PD signal characteristics of mineral oils and vegetable oils in the time and frequency domains, which provide useful information about the condition of the insulating oil. Typical PD pulse (left side) and corresponding frequency spectrum (right 


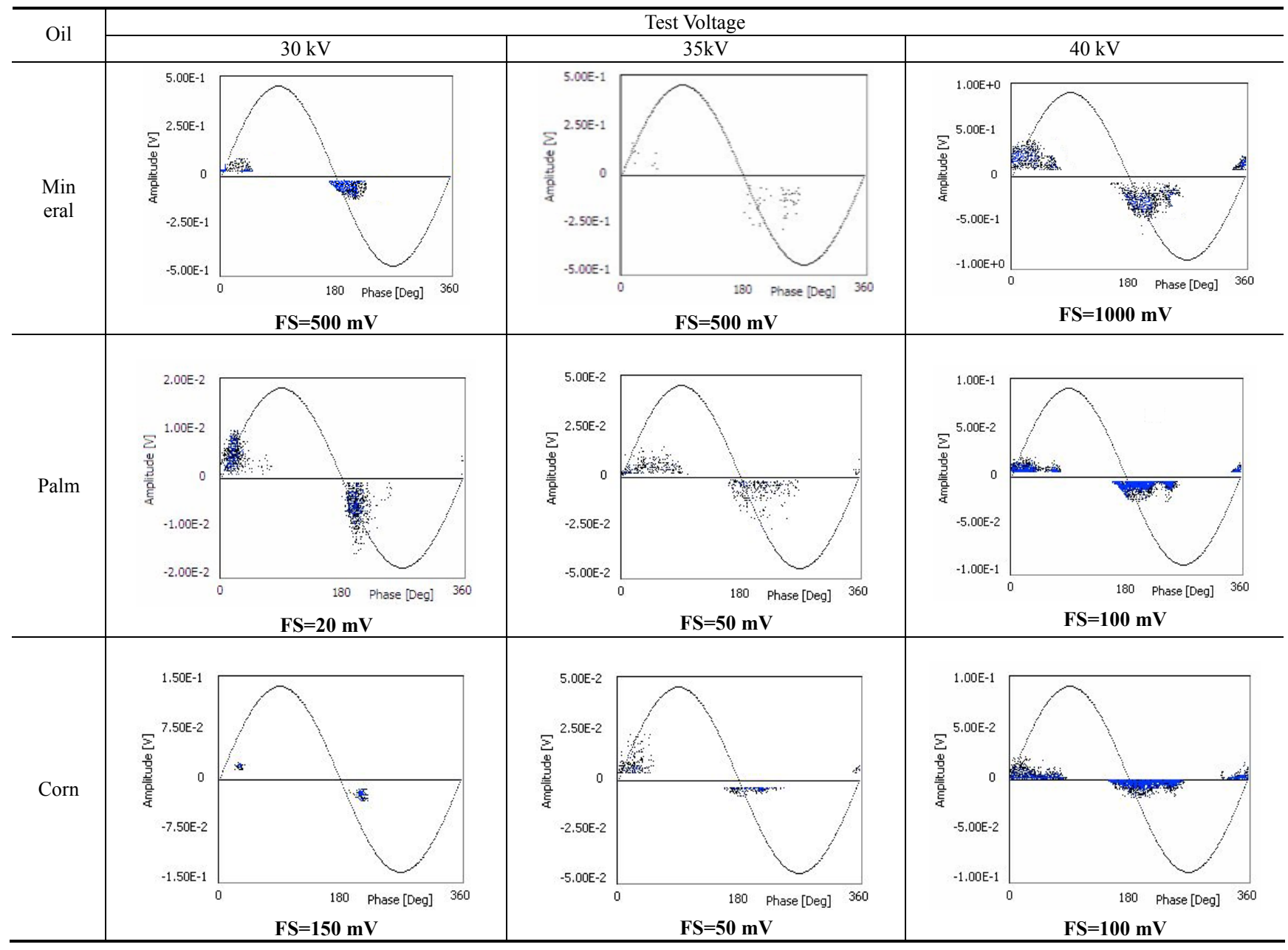

Fig. 9. Typical PRPD pattern for the 30 days thermally aged oil specimens obtained at different voltage levels. FS stands for full scale.

side) obtained at needle-plane electrode configuration of palm oil at different aged conditions are shown in Fig. 10. From the PD pulses, it is observed that with respect to increase in thermal aging, the peak value of PD pulse is considerably increased. From the frequency spectrum of the PD pulses, it is clear that irrespective of the type of aging, three peaks are noticed at 7,20 and $33 \mathrm{MHz}$ respectively. Similar tests were repeated for mineral oil and corn oil and it is noticed that there is no significant difference in PD pulse characteristics at similar experimental and aging conditions.

\subsection{Statistical analysis of PD pattern}

In the case of real time outdoor PD measurements on transformers, successful and accurate insulation condition diagnosis based on PD analysis depends on the collection of statistical information on the PD signals. Reports on the statistical information on the PD characteristics of vegetable oils are scanty. Hence, in the present work, analysis of the stochastic features of PD-pulse sequences at needle-plane electrode configuration was carried out, which can be used as a parameter for understanding aging condition of vegetable oil insulation.

Typical statistical quantities for PD pulse magnitude are skewness and mean value of the shape parameter (Beta) of the Weibull function [17-18]. Skewness is a most commonly used parameter to check how the data are distributed in the given range, whereas shape parameter is associated with the dispersion of PD pulse height. PD instrument software evaluates the shape parameter and skewness values of each PRPD pattern data and the corresponding values were noted down for further analysis.

Fig. 11 shows the mean values of shape parameter, obtained with $95 \%$ confidence intervals from the PD amplitude distribution, at different aging conditions of oils. The corresponding minimum and the maximum values of the same parameter obtained from the database are also reported. In this figure, VIR represents virgin oil, T1 represents 45 days thermal aged oil without copper and T2 represents 45 days thermal aged oil with copper. In general, as the aging severity increases, the reduction in $\beta$ values is noticed. Trend followed by $\beta$ value clearly indicates the increase in data dispersion with increase in aging severity. 

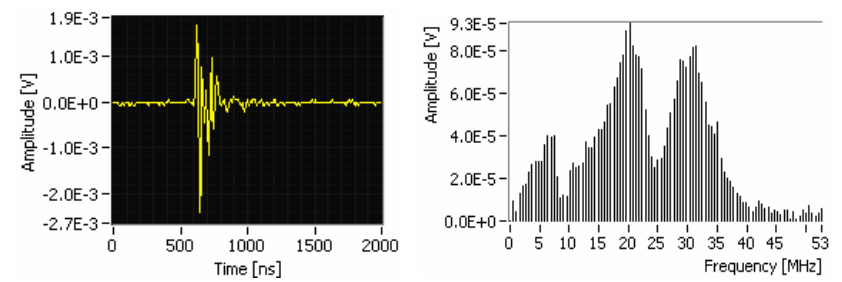

(a)
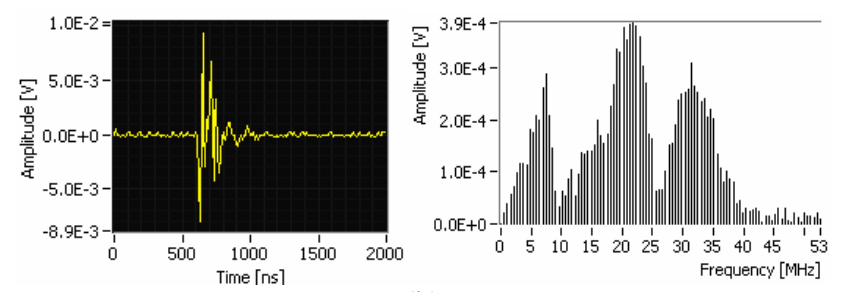

(b)
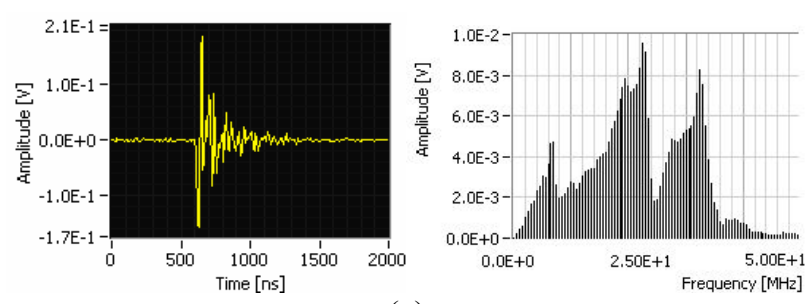

(c)

Fig. 10. Partial discharge pulse (left) and corresponding frequency spectrum (right) obtained at needleplane electrode configuration of palm oil: (a) virgin; (b) 45 days thermal aged without copper; (c) 45 days thermal aged with copper

Vegetable oils showed higher $\beta$ value compared with mineral oil at all aging conditions.

Fig. 12 shows the minimum, maximum and mean values of skewness parameter, obtained with 95 percent confidence intervals from the PD amplitude distribution, at different aging conditions. It is observed that with increase in aging severity, the value of skewness considerably increases, which indicates the reduction in symmetry of the data at thermal aged conditions. In general, from the statistical analysis of PD pattern, it is observed that variation of shape parameter and skewness are closely related to the aging condition of the vegetable oils.

The above reported PD characteristics of palm and corn oil clearly show that they have the ability to be considered as an alternate for mineral oils. Since PD in liquid dielectrics generates from any stress enhancement sites, its reproducibility in discharge measurements is practically difficult to achieve and hence it would be sensible to operate transformers below the PDIV level and thereby prevent possible molecular chain scission and chemical degradation of vegetable oils in the presence of PD.

It is reported in [22] that temperature changes may result in variation of PD properties and hence further research activities are being carried out to understand the influence of elevated temperature on the PD characteristics of these vegetable oils. Also, increase in viscosity of vegetable oils,

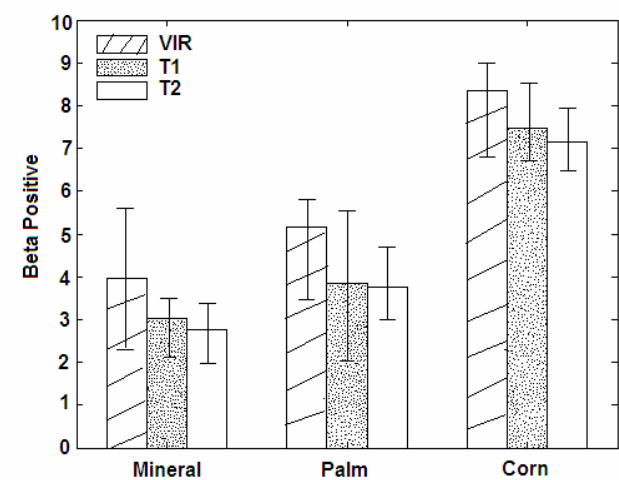

Fig. 11. Variation of shape parameter Beta positive of PD pattern at different aging conditions

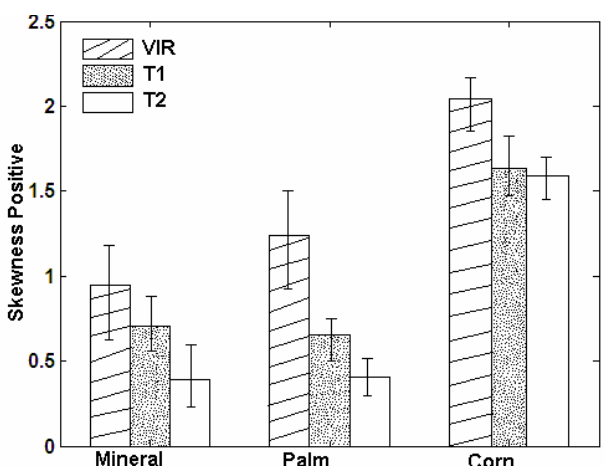

Fig. 12. Variation of skewness positive of PD pattern at different aging conditions

as reported in many research papers, due to increase in aging time is clearly not beneficial for high voltage transformer applications. Hence further research work is being carried out to improve the viscosity property of vegetable oils under long term thermal stress.

\section{Conclusion}

Partial discharge and breakdown strength experiments were performed in the laboratory for both virgin and thermally aged palm and corn oils. Long term thermal aging of palm and corn oil slightly reduces the electrical breakdown strength, however the BDV value lies above mineral oil. Partial discharge inception voltage of palm and corn oil are higher than mineral oil. Presence of copper during aging process significantly increased PD activities in mineral oil and vegetable oils. PD amplitude of palm oil and corn oil are lower than mineral oil with respect to increase in applied voltage and also with respect to increase in thermal stress aging period. Results show that there is no considerable difference in terms of time domain and frequency domain characteristics of PD signals for both vegetable oils and mineral oil considered in this work. From the statistical analysis of PRPD pattern, skewness and shape parameter value considerably reduce with long 
term thermal aging and hence can be used as a measure of insulation aging. Reported results indicate that palm and corn oil has the required potential for high voltage electrical insulation applications and it clearly motivates to carry out further extensive research over these vegetable oils.

\section{Acknowledgements}

Author(S.C) would like to sincerely thank TechIMP, Italy for their support in the partial discharge measurements.

\section{References}

[1] E.Kuffel and W.S. Zaengl, "High Voltage Engineering Fundamentals", Pergamon Press, Oxford, New York, 1984.

[2] J. Li, Z. Zhang, P. Zou, S. Grzybowski, and M. Zahn, "Preparation of a Vegetable Oil-based Nanofluid and Investigation of Its Breakdown and Dielectric Properties", IEEE Electr. Insul. Mag., vol. 28, no. 5, pp. 43-50, 2012.

[3] Suwarno M. Ilyas, "Study on the Characteristics of Jatropha and Ricinnus Seed Oils as Liquid Insulating Materials", in Proceedings of the Annual Report Conference on IEEE Electrical Insulation and Dielectric Phenomena, pp.162-166, 2006.

[4] V.Arun and S.Chandrasekar, "Study on electrical and thermal characteristics of biodegradable vegetable oils for power transformer applications", in Proceedings of the International conference on electrical energy systems and power electronics in emerging economies, ICEESPEEE'09, SRM University, Chennai, pp.1012-1016, 16-17 Apr 2009.

[5] U.U. Abdullahi, S.M. Bashi, Robia Yunus, Mohibullah and Hj. Anlir Nurdin, "The Potentials of Palm Oil as a Dielectric Fluid", in Proc. of National Power \& Energy Conference (PECon), pp. 224-228, Kuala Lumpur, Malaysia, 2004.

[6] Suwarno Aditama, "Dielectric properties of Palm oils as liquid insulating materials: effects of fat content", in Proc. of International symposium on electrical insulating materials, pp. 91-94, Japan, June 2005.

[7] Martin, N. Lelekakis, W. Guo, and Y. Odarenko, "Further Studies of a Vegetable-Oil-Filled Power Transformer", IEEE Electr. Insul. Mag., vol. 27, no. 5, pp. 6-13, 2011.

[8] L. Hosier, A. Guushaa, E.W. Westenbrink, C. Rogers, A. S. Vaughan, and S. G. Swingler, "Aging of Biodegradable Oils and Assessment of Their Suitability for High Voltage Applications", IEEE Trans. Dielectr. Electr. Insul., vol. 18, no. 3, pp. 728-738, 2011.

[9] B. S. H. M. S. Y. Matharage, M. A. R. M. Fernando, M.A.A.P. Bandara, G.A.Jayantha and C.S.Kalpage,
"Performance of Coconut Oil as an Alternative Transformer Liquid Insulation", IEEE Trans. Dielectr. Electr. Insul., vol. 20, no. 3, pp. 887-898, 2013.

[10] J.I. Jeong, Jung-SikAn and C.S. Huh, "Accelerated Aging Effects of Mineral and Vegetable Transformer Oils on Medium Voltage Power Transformers", IEEE Trans. Dielectr. Electr. Insul., vol.19, no.1, pp.156$161,2012$.

[11] Stefan Tenbohlen and Maik Koch, "Aging Performance and Moisture Solubility of Vegetable Oils for Power Transformers", IEEE Trans. Power Delivery, vol.25, no.2, pp.825-830, 2010.

[12] M.H. Abderrazzaq and F. Hijazi, "Impact of Multifiltration Process on the Properties of Olive Oil as a Liquid Dielectric", IEEE Trans. Dielectr. Electr. Insul., vol. 19, no. 5, pp. 1637-1680, 2012.

[13] G.K.Frimpong, T.V.Oommen and R.Asano, “A Survey of Aging Characteristics of Cellulose Insulation in Natural Ester and Mineral Oil", IEEE Electr. Insul. Mag., vol. 27, no. 5, pp. 36-48, 2011.

[14] D. Martin and Z.D. Wang, "Statistical Analysis of the AC Breakdown Voltages of Ester Based Transformer Oils”, IEEE Trans. Dielectr. Electr. Insul., vol. 15, no. 4, pp. 1044-1050, 2008.

[15] Contin, A. Cavallini, G.C. Montanari, G. Pasini and F. Puletti, "Digital detection and fuzzy classification of partial discharge signals", IEEE Trans. Dielectr. Electr. Insul., vol. 9, pp. 335-348, 2002.

[16] Andrea Cavallini, S.Chandrasekar and Gian Carlo Montanari, "Inferring Ceramic Insulator Pollution by an Innovative Approach Resorting to PD Detection", IEEE Trans. Dielectr. Electr. Insul., vol. 14, no. 1, pp. 23-29, Feb 2007.

[17] Andrea Cavallini, A. Contin, Gian Carlo Montanari, and F. Puletti, "Advanced PD inference in on-field measurements. Part I. Noise rejection", IEEE Trans. Dielectr. Electr. Insul., vol. 10, pp. 216-224, 2003.

[18] Cavallini, M. Conti, A. Contin and G.C. Montanari, "Advanced PD Inference in On-Field Measurements. Part.2: Identification of Defects in Solid insulation Systems", IEEE Trans. Dielectr. Electr. Insul., vol. 10, pp. 528-538, 2003.

[19] M. Pompili, "Partial discharge development and detection in dielectric liquid", IEEE Trans. Dielectr. Electr. Insul., vol. 16, no. 6, pp. 1648-1654, 2009.

[20] D. Imamovic, K.X. Lai, N.A. Muhamad, B.T. Phung and T.R. Blackburn, "Partial Discharge and Dissolved Gas Analysis in Bio-Degradable Transformer Oil", in Proc. of Cigre 16, pp. 1-7, Brugge 2007.

[21] Zhongdong Wang, Xin Wang, Xiao Yi and Sitao Li, "Gas Generation in Natural Ester and Mineral Oil Under Partial Discharge and Sparking Faults", IEEE Electrical Insulation Magazine, vol. 29, no. 5, pp. 6270,2013.

[22] W. G. Ariastina, B. T. Phung and T. R. Blackburn, "Measurement of partial discharges in vegetable oilimpregnated insulating system", in Proc. of TENCON IEEE Region 10 Conference, pp. 954-958, 2011. 


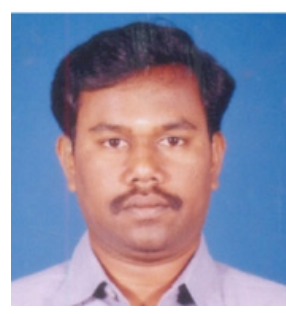

chanics.

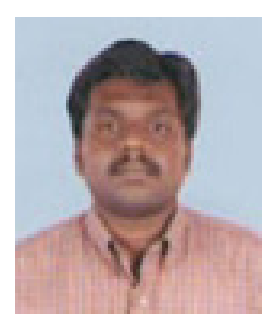

B. Karthik He received B.E degree in Electrical and Electronics Engineering from Annamalai University, India in 2003 and ME Degree in Power Systems from Sona college of technology, India in 2006 and Ph.D degree from Anna University, Chennai in 2013. Presently, he is working as Assistant Professor at Sona College of Technology in the Department of Electrical and Electronics Engineering. His area of research interest is power systems and soft computing techniques.

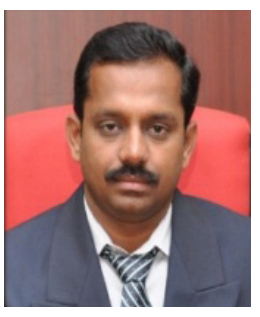

S. Chandrasekar He received the B.E. degree in Electrical and Electronics Engineering from Thiagarajar college of Engineering, Madurai in 1996 and M.E degree in Power Systems from Coimbatore Institute of Technology, Coimbatore in India in 2001 and the $\mathrm{Ph} . \mathrm{D}$ degree from Indian Institute of Technology Madras, India in 2005. He was a postdoctoral research fellow at the University of Bologna, Italy from 2005 to 2006. Currently, he is working as a Professor at Gnanamani College of Technology. His area of research interest is electric power engineering and soft computing techniques. 